\title{
A New Concept for the Treatment and Rehabilitation of Patients with Pathologic Comorbidities Using Cutting-Edge Digital Technologies in Dental Orthopaedics
}

\author{
Alexey V. Yumashev ${ }^{1}$ Maria V. Mikhailova ${ }^{1} \quad$ Igor V. Fomin ${ }^{1} \quad$ Jiaqi Li $^{1} \quad$ Bowen Yang ${ }^{1}$ \\ ${ }^{1}$ Department of Prosthetic Dentistry, I.M. Sechenov First Moscow \\ State Medical University (Sechenov University), Moscow, Russia \\ Address for correspondence Alexey Yumashev, MD, Department \\ of Prosthetic Dentistry, I.M. Sechenov First Moscow State Medical \\ University (Sechenov University), 8-2 Trubetskaya Street, \\ Moscow 119992, Russia, (e-mail: yumashev031996@mail.ru).
}

\begin{abstract}
Keywords

- dentistry

- orthopaedic treatment

- adentia

- dentophobic reactions

Objectives This article investigates the specific aspects of overactive or exaggerated vomiting reflexes affecting the procedure of dental examination and impression in patients with complete secondary adentia, who need orthopaedic dental treatment.

Materials and Methods The prevailing manifestation degree of exaggerated vomiting reflex was diagnosed among patients with complete secondary adentia and exaggerated vomiting reflex.

Statistical Analysis Exaggerated vomiting reflexes occur when a patient suffers from dentophobia, the term, which is otherwise known as the fear of dentists. The study was performed using methods of mathematical statistics, including the Pearson $\chi^{2}$ criterion and the statistical probability criterion $(p)$.

Results The role of an exaggerated vomiting reflex was revealed in the development of patients' dentophobic experiences, and the nature of such experiences was established. Variations in dentophobic reactions were distinguished and management strategies were studied for patients with complete secondary adentia and exaggerated vomiting reflex.

Conclusions These studies were aimed at preventing the development of vomiting reflex during dental procedures and at identifying an optimal strategy for stopping exaggerated vomiting reflex. The role of the vomiting reflex in the orthopaedic treatment of dental patients was determined.
\end{abstract}

\section{Introduction}

The relevance of improving dental care in Russia is determined by the significant prevalence of dental pathology, reaching 70 to $100 \%$ in various regions. ${ }^{1-3}$ Various types of adentia occupy oral diseases, and in this regard, the focus of scientific medical research is aimed at improving the provision of medical care for this category of patients. ${ }^{3-6}$ Modern dentistry pays much attention to gender and gerontology. ${ }^{7.8}$ Additionally, such factors as the vomiting reflex in dental patients often remain outside the attention of researchers, and only rare publications are devoted to this problem. ${ }^{79,10}$

Nevertheless, dentists often face the necessity to provide orthopaedic dental care to patients of various age groups with adentia and exaggerated vomiting reflex., ${ }^{4,11,12}$ It is known that the exaggerated vomiting reflex can be one of the factors causing deterioration of dental health by provoking dentophobic reactions in patients. Discomfort while visiting a dentist and experiencing oral sanitation can result in the patient's rejection of dental interventions and, as a 
consequence, in the progression of dental pathology with the subsequent development of adentia, as well as it can negatively affect the psychological health of patients. ${ }^{13-15}$

In cases when patients with adentia and exaggerated vomiting reflexes seek dental care, it is essential to determine the optimal treatment strategy. ${ }^{12,16,17}$ Currently, a two-stage intraosseous dental implantation using intraoral scanning to simplify the individual stages of denture manufacturing is the most widespread modern method for the orthopaedic treatment of secondary adentia. ${ }^{18-21}$

However, without a special modification of the dental examination procedure and orthopaedic treatment aimed at preventing vomiting reflex activation, it is often difficult to perform dental procedures for patients with exaggerated vomiting reflexes. For this purpose, various methods are applied: from introducing elements of psychotherapy in dental practice to employing digital impressions. , $8,17,22,23^{2}$

The above-mentioned method makes us pay more attention to the specific aspects of the impact of exaggerated vomiting reflexes on dental interventions and predetermines the search for ways to prevent and mitigate the adverse consequences of exaggerated vomiting reflexes in dental patients. The purpose of the research is to establish the peculiarities of the exaggerated vomiting reflex impact while conducting dental examination and getting dental impressions for patients with complete secondary adentia seeking orthopaedic dental treatment.

\section{Materials and Methods}

This was a clinical, prospective single-center randomized controlled trial. The study was conducted at the Department of Orthopaedic Dentistry at the I.M. Sechenov First Moscow State University, based on the Maxillofacial Surgery Clinic of the I.M. Sechenov First Moscow State University (Moscow, Russia). Two hundred patients with complete secondary adentia requiring dental orthopaedic treatment were examined and treated. All patients would have a jaw impression during medical procedures. There were 108 (54\%) women and $92(46 \%)$ men. Both sexes were represented in the sample in relatively equal proportions $(p>0.05)$. The age of the patients ranged from 41 to 68 years; the average age of the patient was $53.72 \pm 2.06$ years.

The study consisted of two stages. At the first stage, the patients were examined to find out the severity of the vomiting reflex and the peculiarities of psychological experiences due to the vomiting reflex. At the second stage of the study, the patients were treated with various tactics of stopping the vomiting reflex during the procedure of dental examination and imprinting procedure. The patients were divided into two groups according to the purpose of the research.

The first group was the experimental group (EG) that included 98 patients with the exaggerated vomiting reflex and complete secondary adentia, who needed orthopaedic treatment. This group was divided into three subgroups depending on the prophylactic management strategies that were applied and aimed at reducing the severity of vomiting reflex manifestation during dental procedures. The EG-1.1 subgroup included 33 patients for whom topical spray anesthesia with $10 \%$ lidocaine solution was prescribed before taking the dental impression. The EG-1.2 subgroup included 32 patients who received $0.1 \%$ Galazolin nasal solution 1 to 3 minutes before the dental impression was taken. The EG-1.3 subgroup consisted of 33 patients who were subjected to an "optical impression" using the 3Shape Trios intraoral scanner to reduce vomiting reflex manifestations instead of classical imprinting.

The second group was the control group (CG) and included 102 patients with complete secondary adentia seeking orthopaedic treatment. No exaggerated vomiting reflex was noted in these patients, and, therefore, standard dental examination and impression taking strategies were applied. There were no statistically significant gender or age differences between the experimental and the CG $(p>0.05)$.

The research methods included dental history, clinical history, and stomatoscopic impression taking methods. Diagnostic measures were aimed at eliminating factors that impede rational prosthetics, such as the presence of congenital or acquired pathological, morphological and functional conditions of the bone-maxillary apparatus, diseases and pathological changes in the tissue of the oral cavity, exostoses, tumor-like diseases, and the establishment of the actual dental status. The impressions were taken by transfer copying using the open or closed tray technique, depending on the available conditions, and by means of the 3Shape Trios intraoral scanning.

The novelty of this research is the use of minimally invasive intraoral scanner 3Shape Trios to prevent not only the vomiting reflex but also dentophobic reactions in patients with increased vomiting reflex during the scratching print procedure. Both methods, such as the method of comparing minimally invasive scanner and pharmacological medications, can reduce the vomiting reflex, but it was been investigated how they affect the psychological well-being and dentophobic reactions in patients with then increased vomiting reflex. Statistical analysis was performed using methods of mathematical statistics, including the Pearson $\chi^{2}$ criterion and the statistical probability criterion $(p)$.

\section{Results}

The degree of vomiting reflex manifestation among patients with the exaggerated vomiting reflex shifted toward the moderately expressed exaggerated vomiting reflex (43 patients-43.88\%). Moderately expressed exaggerated vomiting reflexes were diagnosed in 32 (32.65\%) EG patients. The number of patients with strongly and very strongly expressed vomiting reflexes did not exceed $1 / 3$ of the number of patients in the EG group and amounted to 12 and 15 people (11.22 and $14.29 \%$ ), respectively. In the CG, all patients were diagnosed with a minimal degree of vomiting reflex manifestation (-Table 1). Tongue root, distal portion of the hard palate, soft palate, and mandibular retromolar areas served as anatomical landmarks of vomiting reflex trigger zones in the oral cavity

In $97.96 \%$ of observations in the EG, the available exaggerated vomiting reflexes were associated with dentophobic experiences and several dentophobic reactions, which 
Table 1 The degrees of gag reflex manifestation in patients with complete secondary adentia

\begin{tabular}{|c|c|c|c|c|c|}
\hline \multirow{3}{*}{$\begin{array}{l}\text { Degree of } \\
\text { gag reflex } \\
\text { manifestation }\end{array}$} & \multirow[t]{3}{*}{ Gag reflex characterization } & \multicolumn{4}{|c|}{ Clinical trial groups } \\
\hline & & \multicolumn{2}{|c|}{$\begin{array}{c}\text { EG } \\
(n=98)\end{array}$} & \multicolumn{2}{|c|}{$\begin{array}{c}\text { CG } \\
(n=102)\end{array}$} \\
\hline & & $n$ & $\%$ & $n$ & $\%$ \\
\hline 1 & $\begin{array}{l}\text { Normal vomiting reflex. Rare, patient-controlled } \\
\text { attacks }\end{array}$ & - & - & 102 & 100 \\
\hline 2 & $\begin{array}{l}\text { Mild exaggerated vomiting reflex. It is necessary } \\
\text { to control and prepare the patient for dental } \\
\text { intervention }\end{array}$ & 32 & 32.65 & - & - \\
\hline 3 & $\begin{array}{l}\text { Moderate exaggerated vomiting reflex. The vomiting } \\
\text { reflex limits the duration of individual procedures } \\
\text { and complicates the use of various techniques in } \\
\text { treatment }\end{array}$ & 43 & 43.88 & - & - \\
\hline 4 & $\begin{array}{l}\text { Strong vomiting reflex. It is impossible for the patient } \\
\text { to visit a dentist without special measures. The } \\
\text { vomiting reflex can occur even during the oral cavity } \\
\text { examination }\end{array}$ & 11 & 11.22 & - & - \\
\hline 5 & $\begin{array}{l}\text { Very strong vomiting reflex. Dental treatment is } \\
\text { impossible without the use of means to reduce the } \\
\text { vomiting reflex }\end{array}$ & 14 & 14.29 & - & - \\
\hline & $\chi^{2}$ & \multicolumn{2}{|c|}{37.05} & \multicolumn{2}{|c|}{204.00} \\
\hline & $p$-Value & \multicolumn{2}{|c|}{$<0.001$} & \multicolumn{2}{|c|}{$<0.001$} \\
\hline
\end{tabular}

significantly differed from the CG results, where dentophobic experiences and the resulting dentophobic reactions were observed in $36.27 \%$ of observations.

The nature of dentophobic experiences was diverse and included a wide range of contents-from fear of pain and helplessness during medical procedures to fear of dental medical instruments. In $73.47 \%$ of cases, dentophobic experiences were directly related to exaggerated vomiting reflexes, and included phobias such as fear of vomiting during dental procedures, choking on vomit due to the vomiting reflex activation during dental procedures, contaminating clothes or floor with vomit, and jaw clenching due to the vomiting reflex activation in the process of dental care provision followed by a bite of dental instruments ( - Table 2 ).

Dentophobic reactions, in addition to the vegetative and emotional states, included a verbal component, macro- and micromotor responses in the form of tilted body, head turns, jaw clenching, increased swallowing movements, etc., which complicate dental examination and obtaining a high-quality impression. Without taking preventive measures aimed at reducing the vomiting reflex, dental procedures in all EG patients were difficult or impossible to conduct.

To prevent the vomiting reflex development in the EG patients, one of the following preventive strategies was implemented:

- Using topical spray anesthesia with $10 \%$ lidocaine solution prior to impression taking;

- Using $0.1 \%$ nasal solution of Galazolin 1 to 3 minutes prior to impression taking;

- Using the 3Shape Trios intraoral scanner, which has a function of double scanning to create a three-dimensional image of gingiva and prosthetic bed.
The EG patients were divided into three subgroups (EG-1.1, EG-1.2, EG-1.3), which did not have significant differences among themselves, to study the effectiveness of measures to prevent the vomiting reflex activation. One of the above preventive tactics was applied to these patients; the results are given in - Table 3. It was established that the topical application of $10 \%$ lidocaine solution spray enabled to reduce vomiting reflexes; however, the use of this medication provoked the development of unpleasant sensations in patients, such as throat irritation, hypersalivation, swollen mucosa in distal portions of the hard palate, a feeling of slight suffocation with restless behavior, and micromotions when the impression was taken. The indicated adverse reactions during impression taking by this method-using $10 \%$ lidocaine solution spray-led to inaccurate impressions, which subsequently caused insufficient adherence of complete dentures and an increase in the denture adaptation time. Upon completion of impression taking, until the effect of the medication was terminated, patients complained of dysphagia, the excessive saliva accumulation in the mouth, the preserved feeling of slight suffocation, and a fear of choking on saliva.

The use of $0.1 \%$ Galazolin nasal solution to prevent vomiting reflex activation caused less unpleasant sensations in patients during dental care provision. While the impression mass was kept for the required time in the oral cavity, the researchers identified a lack of excessive salivation vomiturition and restless behavior of patients, which promoted the good quality of the impressions. However, upon completion of dental procedures $12.50 \%$ of patients complained of oral and nasal dysphagia and dizziness.

The use of the 3Shape Trios scanner for impression taking caused less dentophobic experiences in patients with exaggerated vomiting reflexes. The scanner enabled to obtain a high-quality "optical impression" with the ability to 
Table 2 The nature of dentophobic experiences of patients with complete secondary adentia and exaggerated gag reflex

\begin{tabular}{|c|c|c|c|c|c|c|}
\hline \multirow{3}{*}{$\begin{array}{l}\text { Content of patients' dentophobic } \\
\text { experiences }\end{array}$} & \multicolumn{4}{|c|}{ Clinical trial groups } & \multirow[t]{3}{*}{$\chi^{2}$} & \multirow[t]{3}{*}{$p$-Value } \\
\hline & \multicolumn{2}{|c|}{$\begin{array}{c}\text { EG } \\
(n=98)\end{array}$} & \multicolumn{2}{|c|}{$\begin{array}{c}\text { CG } \\
(n=102)\end{array}$} & & \\
\hline & $n$ & $\%$ & $n$ & $\%$ & & \\
\hline Fear of dental pain & 96 & 97.96 & 37 & 36.27 & 85.37 & $<0.001$ \\
\hline Fear of dentists & 1 & 1.02 & 2 & 1.96 & 0.30 & $>0.05$ \\
\hline Fear of the dental office & 0 & 0.00 & 1 & 0.98 & 0.97 & $>0.05$ \\
\hline Fear of dental medical instruments & 42 & 42.86 & 22 & 21.57 & 10.41 & 0.002 \\
\hline Fear of dental procedures & 70 & 71.43 & 35 & 34.31 & 27.61 & $<0.001$ \\
\hline $\begin{array}{l}\text { Fear of helplessness during medical } \\
\text { procedures }\end{array}$ & 59 & 60.20 & 3 & 2.94 & 76.62 & $<0.001$ \\
\hline $\begin{array}{l}\text { Fear of choking on saliva during dental } \\
\text { procedures }\end{array}$ & 72 & 73.47 & 8 & 7.84 & 89.69 & $<0.001$ \\
\hline $\begin{array}{l}\text { Fear of a foreign body aspiration during dental } \\
\text { procedures }\end{array}$ & 30 & 30.61 & 10 & 9.80 & 13.53 & $<0.001$ \\
\hline Fear of vomiting during dental procedures & 72 & 73.47 & - & - & 117.09 & $<0.001$ \\
\hline $\begin{array}{l}\text { Fear of choking on vomit due to gag reflex } \\
\text { activation during dental procedures }\end{array}$ & 72 & 73.47 & - & - & 117.09 & $<0.001$ \\
\hline $\begin{array}{l}\text { Fear of contaminating clothes or floor with } \\
\text { vomit in case of gag reflex activation during } \\
\text { dental procedures }\end{array}$ & 18 & 18.37 & - & - & 20.59 & $<0.001$ \\
\hline $\begin{array}{l}\text { Fear of jaw clenching due to gag reflex acti- } \\
\text { vation in the process of dental care provision } \\
\text { followed by a bite of dental instruments }\end{array}$ & 36 & 36.73 & - & - & 45.69 & $<0.001$ \\
\hline Total patients with dentophobic experiences & 96 & 97.96 & 37 & 36.27 & 85.37 & $<0.001$ \\
\hline
\end{tabular}

Abbreviations: EG, experimental group; CG, control group.

Table 3 Comparative characteristics of the effectiveness of prophylactic management strategies for patients with complete secondary adentia and exaggerated gag reflex

\begin{tabular}{|c|c|c|c|c|c|c|c|c|}
\hline \multirow[t]{3}{*}{ Indicator } & \multicolumn{6}{|c|}{ EG subgroups } & \multirow[t]{3}{*}{$\chi^{2}$} & \multirow[t]{3}{*}{$p$-Value } \\
\hline & \multicolumn{2}{|c|}{$\begin{array}{c}\text { EG-1.1 } \\
(n=33)\end{array}$} & \multicolumn{2}{|c|}{$\begin{array}{c}\text { EG-1.2 } \\
(n=32)\end{array}$} & \multicolumn{2}{|c|}{$\begin{array}{c}\text { EG-1.3 } \\
(n=33)\end{array}$} & & \\
\hline & $n$ & $\%$ & $n$ & $\%$ & $n$ & $\%$ & & \\
\hline \multicolumn{9}{|l|}{$\begin{array}{l}\text { Available subjective } \\
\text { sensations: }\end{array}$} \\
\hline -Throat irritation & 17 & 51.52 & 5 & 15.63 & 2 & 6.06 & 20.45 & $<0.001$ \\
\hline -Choking sensation & 10 & 30.30 & 1 & 3.13 & - & - & 18.33 & $<0.001$ \\
\hline -Dizziness & - & - & 4 & 12.50 & - & - & 8.60 & 0.014 \\
\hline -Nausea, vomiturition & 17 & 51.52 & 2 & 6.25 & - & - & 33.27 & $<0.001$ \\
\hline -Hypersalivation & 16 & 48.48 & - & - & - & - & 37.66 & $<0.001$ \\
\hline -Xerostomia & - & - & 4 & 12.50 & - & - & 8.60 & 0.014 \\
\hline -Discomfort in the oral cavity & 18 & 54.55 & 12 & 37.50 & 4 & 12.12 & 13.27 & 0.002 \\
\hline $\begin{array}{l}\text { Impression taking is } \\
\text { impossible }\end{array}$ & 2 & 6.06 & - & - & - & - & 4.02 & 0.134 \\
\hline $\begin{array}{l}\text { Impression taking is } \\
\text { complicated }\end{array}$ & 10 & 30.30 & 7 & 21.88 & - & - & 11.25 & 0.004 \\
\hline $\begin{array}{l}\text { Impression taking is without } \\
\text { complications }\end{array}$ & 11 & 33.33 & 25 & 78.13 & 33 & 100.00 & 36.56 & $<0.001$ \\
\hline \multicolumn{9}{|l|}{ Impression quality: } \\
\hline -Unsatisfactory & 5 & 15.15 & 2 & 6.25 & - & - & 5.77 & 0.056 \\
\hline -Satisfactory & 26 & 78.79 & 30 & 93.75 & 33 & 100.00 & 9.39 & 0.010 \\
\hline
\end{tabular}

Abbreviation: EG, experimental group. 
interrupt scanning for the patient to have a rest in the event of the vomiting reflex; there were no adverse effects of the procedure, which allows us to consider this strategy as a preferred one for impression taking in patients with exaggerated vomiting reflexes.

\section{Discussion}

All researchers agree that patients with increased vomiting reflex need a correction of therapy to avoid the unpleasant consequences of the reflex during dental procedures. However, there is still a question which therapeutic tactics aimed at eliminating the increased vomiting reflex is the most effective. $911,14,19,23$

In this study, the authors applied three prophylactic tactics to patients aimed at reducing the severity of the vomiting reflex during dental procedures. Since previous researchers point to the positive effect of short-term pharmacotherapy for eliminating the increased vomiting reflex,9 two of the tactics we used were pharmacotherapy - using local aerosol anesthesia with $10 \%$ lidocaine solution and $0.1 \%$ Galazolin nasal solution, and the third tactic was instrumental - 3Shape Trios intraoral scanner. We expected that the use of an intraoral scanner will help improve the condition of patients with an increased emetic reflex since this tactic excludes the presence of triggers for activating the vomit reflex.

Many researchers point out that psychotherapy is an effective tactic that helps to cure increased vomiting reflex.9 The authors did not include psychotherapy in the study since it is significantly time-consuming and involves involving not only a dentist but also a psychotherapist in therapy. Such a tactic for the treatment of increased vomiting reflex as acupuncture, which is based on the studies by Fiske J. and Dickinson C. It eliminates or partially eliminates the increased vomiting reflex,11 the authors excluded from the study since acupuncture is not recognized in all countries by classical medicine.

This study demonstrated that, although all three tactics allowed for reducing the severity of the gag reflex, the effectiveness of these tactics is different. Two of the tactics used - the use of local aerosol anesthesia with $10 \%$ lidocaine solution and $0.1 \%$ Galazoline nasal solution had side effects that made it difficult to obtain a high-quality impression, such as nausea, vomiting, dizziness, hypersalivation, or vice versa, dry mucous membranes. The authors believe that this is because the side effects of these tactics are identical to those effects that accompany the increased vomiting reflex and its consequences, and therefore provoke the appearance of the same stomatophobic reactions in patients as with the activation of the vomiting reflex.

When using the 3Shape Trios intraoral scanner, several patients also reported side effects in the form of an unpleasant sensation in the mouth or a sore throat, but this did not lead to problems when taking an impression.

In addition, not all of these tactics made it possible to eliminate completely the stomatophobic reactions associated with an increased emetic reflex, which also affected the quality of the obtained impression. So, satisfactory quality of casts in all patients could be obtained only with the use of the 3Shape Trios intraoral scanner; when applying local aerosol anesthesia with a $10 \%$ solution of lidocaine or a $0.1 \%$ nasal solution of Galazolin in $15.15 \%$ and $6.25 \%$, the quality of the casts was unsatisfactory due to the destructive behavior of patients due to stomatophobic reactions.

This study proved the effectiveness of using the 3Shape Trios intraoral scanner to take a high-quality impression in patients requiring prosthetics and suffering from an increased vomiting reflex. However, its effectiveness in other dental procedures in patients with an increased gag reflex is debatable and requires additional research.

\section{Conclusions}

The role of the vomiting reflex in orthopaedic treatment of dental patients is reduced to the following aspects:

- The development of dentophobic experiences associated with the available exaggerated vomiting reflex and dentophobic reactions in patients prior to and/or during dental procedures;

- The development of motor-behavioral reactions due to the vomiting reflex activation during the provision of dental care;

- The need to modify the dentist's behavior before starting medical procedures toward joining a time-consuming explanatory health counseling aimed at stopping the dentophobic experiences conditioned by the exaggerated vomiting reflex.

In general, the available exaggerated vomiting reflexes in patients seeking orthopaedic treatment lead to an increase in the time of dental examination, an expansion of the range of dental procedures with the need to attract additional pharmacological preparations or dental instruments; the above determined the need to update the problem of exaggerated vomiting reflexes in dental practice and to develop preventive medical measures.

\section{Conflict of Interest}

None declared.

\section{References}

1 Avdeenko O, Novikova I, Turkina A, Makeeva I. Oral behavior and dental status of orthodox Christian priests and monks. J Int Soc Prev Community Dent 2019;9(2):137-143

2 Bilhan H, Erdogan O, Ergin S, Celik M, Ates G, Geckili O. Complication rates and patient satisfaction with removable dentures. J Adv Prosthodont 2012;4(2):109-115

3 Furtado Á, Furtado GC, El Haje O, et al. Soft-tissue cone-beam computed tomography (ST-CBCT) technique for the analysis of skeletal, dental and periodontal effects of orthopedic rapid maxillary expansion. J Clin Exp Dent 2018;10(9):e883-e890

4 Fiske J, Dickinson C. The role of acupuncture in controlling the gagging reflex using a review of ten cases. Br Dent J 2001;190(11):611-613

5 Londono J, Abreu A, Baker PS, Furness AR. Fabrication of a definitive obturator from a 3D cast with a chairside digital scanner for a patient with severe gag reflex: a clinical report. J Prosthet Dent 2015;114(5):735-738 
6 Tallarico M, Caneva M, Baldini N, et al. Patient-centered rehabilitation of single, partial, and complete edentulism with cemented- or screw-retained fixed dental prosthesis: The First Osstem Advanced Dental Implant Research and Education Center Consensus Conference 2017. Eur J Dent 2018;12(4):617-626

7 Francetti L, Agliardi E, Testori T. Romeo D, Taschieri S, Del Fabbro M. Immediate rehabilitation of the mandible with fixed full prosthesis supported by axial and tilted implants: interim results of a single cohort prospective study. Clin Implant Dent Relat Res 2008;10(4):255-263

8 Sevbitov AV, Mitin NE, Kuznetsova MYu, Tikhonov VE, Kamenskov PE, Kuznetsov II. Determination of the psychoemotional status of the patients depending on the anomalies of teeth position and bite. Indo Am J Pharmaceutic Sci 2019;6(3):5710-5713

9 Bassi GS, Humphris GM, Longman LP. The etiology and management of gagging: a review of the literature. J Prosthet Dent 2004;91(5):459-467

10 Paszynska E, Dutkiewicz A, Osinska A, et al. Anorexia nervosa with vomiting episodes: dermatological and oral complications. Eur J Dent 2020;14(1):180-185

11 Ershov KA, Sevbitov AV, Dorofeev AE, Pustokhina IG. Evaluation of elderly patients adaptation to removable dentures. Indo Am J Pharmaceutic Sci 2018;5(3):1638-1641

12 Mansour M, Sanchez E, Machado C. The use of digital impressions to fabricate tooth-supported partial removable dental prostheses: a clinical report. J Prosthodont 2016;25(6): 495-497

13 Castro MM, Ferreira RO, Fagundes NCF, Almeida APCPSC, Maia LC, Lima RR. Association between psychological stress and periodontitis: a systematic review. Eur J Dent 2020;14(1):171-179

14 Neumann JK, McCarty GA. Behavioral approaches to reduce hypersensitive gag response. J Prosthet Dent 2001; 85(3):30510.1067/mpr.2001.114273
15 Wismeijer D, Mans R, van Genuchten M, Reijers HA. Patients' preferences when comparing analogue implant impressions using a polyether impression material versus digital impressions (Intraoral Scan) of dental implants. Clin Oral Implants Res 2014;25(10):1113-1118

16 Robinson E. Introducing an alternative drug-free technique for pain and anxiety control into a clinical environment cognitive behavioural therapy: a discussion on implementation into dental practice. SAAD Dig 2014;30:20-24

17 Sevbitov AV, Dorofeev AE, Davidiants AA, Ershov KA, Timoshin AV. Assessment of pain perception of elderly patients with different levels of dentophobia during surgical dental appointment. Asian J Pharmaceutics 2018;12(3):S1012-S1016

18 Kattadiyil MT, Mursic Z, AlRumaih H, Goodacre CJ. Intraoral scanning of hard and soft tissues for partial removable dental prosthesis fabrication. J Prosthet Dent 2014;112(3):444-448

19 Kohavi D. Complications in the tissue integrated prostheses components: clinical and mechanical evaluation. J Oral Rehabil 1993;20(4):413-422

20 Moore R, Brødsgaard I, Rosenberg N. The contribution of embarrassment to phobic dental anxiety: a qualitative research study. BMC Psychiatry 2004;4:1010.1186/1471-244X-4-10

21 Wu J, Li Y, Zhang Y. Use of intraoral scanning and 3-dimensional printing in the fabrication of a removable partial denture for a patient with limited mouth opening. J Am Dent Assoc 2017;148(5):338-341

22 Chutchalermpan T, Pumklin J, Piyapattamin T. Evaluation of disclusion time in various angle's malocclusions by T-scan III system. Eur J Dent 2019;13(4):510-513

23 Kerstein RB. Combining technologies: a computerized occlusal analysis system synchronized with a computerized electromyography system. Cranio 2004;22(2):96-109 